Revista de Investigaciones. Universidad de Nariño -UNED. Vol. VII, No. 1. (ISSN: 0121-120X). (pp. 71-77). 1995.

APA citation style: Benavides B., Jorge E. (1995). Desarrollo del Vocabulario en Inglés Mediante el Uso del Computador en Estudiantes de la Universidad de Nariño. Revista de Investigaciones, Universidad de Nariño, 7(1), 113-126.

\title{
Desarrollo de Vocabulario en Inglés Mediante el Uso del Computador en Estudiantes de la Universidad de Nariño ${ }^{1}$
}

\author{
Jorge E. Benavides B. (joelbebu@gmail.com) \\ Departamento de Lingüística e Idiomas \\ Universidad de Nariño, San Juan de Pasto, Colombia
}

\begin{abstract}
Este estudio de tipo pre-experimental proporciona evidencia inicial sobre la incidencia de uso del computador en el aprendizaje de vocabulario en inglés mediante el uso del computador como parte de una actividad de lectura y reconstrucción de texto. El estudio se realizó durante el primer semestre de 1994 con los estudiantes de segundo semestre del programa de Licenciatura en Informática en las instalaciones de la sala de computadores de la Universidad de Nariño. Después del tratamiento con el computador los sujetos mostraron una diferencia significativa en cuanto al aprendizaje inicial (reconocimiento) de vocabulario. Se utilizaron cinco sesiones diferentes tantas como la presentación de textos en el computador como en la presentación de textos similares en papel y se hicieron pruebas de complementación inmediatamente después de cada sesión. El estudio muestra ganancias significativas en cuanto al aprendizaje inicial de vocabulario utilizando el computador y consecuentemente se insinúa que su incidencia refleja una orientación pedagógica. Los resultados de rendimiento de los sujetos también se dan con los de observación en los que se puede concluir que la interacción sujeto- sujeto puede incidir significativamente en la relación sujeto-máquina en cuanto a aprendizaje se refiere. Esto puede determinar en el futuro la importancia de actividades de tipo complementarias (antes, durante y después) del trabajo con el computador que conllevarían a la diferencia en el aprendizaje.
\end{abstract}

\section{INTRODUCCIÓN AL PROBLEMA}

Durante las dos últimas décadas se ha venido implementando cierta clase de instrucción asistida por computador (IAC) en el área de la educación con resultados de tipo anecdótico

\footnotetext{
${ }^{1}$ Trabajo de investigación presentado como ponencia en el XIV Simposio Anual de la Enseñanza del Inglés. Centro ColomboAmericano. Santafé de Bogotá, Mayo 20-21, 1994.
} 
Revista de Investigaciones. Universidad de Nariño -UNED. Vol. VII, No. 1. (ISSN: 0121-120X). (pp. 71-77). 1995.

sin tener mucha base empírica o que sea el futuro de una investigación de tipo sistemático y consistente.

En el capo del aprendizaje de idiomas por medio de computador y más concretamente en lo que constituye la Informática Educativa para el aprendizaje de los idiomas (INEDAI) han sido muy esperados los estudios de aplicación de software educativo con el propósito de introducirlo o integrarlo a un determinado currículo (Benavides, 1993). Sin embargo, y a pesar del entusiasmo de la gente que impulsa el uso de las nueva tecnologías en Educación, ha sido muy aislado el intento por el cual una implementación y aplicación de los nuevos materiales computarizados en el aprendizaje de los idiomas como el Inglés como lengua extranjera se pueda lograr adecuadamente por el carecer de esta muy importante etapa inicial: la investigación de campo.

Es preocupante ver como en nuestro medio un $40 \%$ de los colegios de nivel bachillerato poseen salas o laboratorios de computadores y sin embargo no saben que uso darles a nivel educativo si no que lastimosamente estos aparatos y los usuarios (en la mayoría de los casos, estudiantes) terminan dándole un uso inadecuado y limitante desde el punto de vista educativo. Existe pues un subutilización de estos equipos cuya adquisición parece ser el fruto de la "moda Informática", el síndrome de la "sala, de informática" sin que medie una planeación e introducción sistemática.

Hay necesidad por consiguiente de iniciar, aunque en forma muy incipiente una clase de estudios exploratorios a nivel cuantitativo y cualitativo sobre las bondades, ventajas y también desventajas de los computadores aplicados al aprendizaje de los idiomas como una posible muestra de la incidencia de la tecnología informática en educación sobre todo en el desarrollo de, supuestamente, nuevas estrategias de aprendizaje. De esta manera se deja de basar los muy comentados poderes de los computadores en el campo educativo en informes, muchas veces, de índole mercantilista o en experiencias anecdóticas.

\subsection{Propósito}

Este estudio de carácter semi-experimental piloto (intrasujetos) pretende explorar a nivel cuantitativo la incidencia de una forma de utilización del computador mediante la aplicación de una clase de materiales informáticos (software educativo) en el desempeño del aprendizaje de vocabulario mediante la lectura en estudiantes de segundo semestre del programa de Licenciatura en informática de la Universidad de Nariño.

Por otro lado se pretende obtener información, mediante observación directa, acerca de las reacciones de los estudiantes al enfrentar una actividad recreada mediante el computador en la que se presupone hay una interacción de máquina y usuario y usuario-usuario al mismo tiempo. 
Revista de Investigaciones. Universidad de Nariño -UNED. Vol. VII, No. 1. (ISSN: 0121-120X). (pp. 71-77). 1995.

\subsection{Hipótesis y Cuestionamientos}

Una clase de hipótesis es el objetivo de comprobación en este estudio. Está relacionada con la incidencia del uso del computador mediante un programa de reconstrucción de texto en el aprendizaje vocabulario en inglés como parte de una actividad de lectura.

Formalmente se considera la clase de hipótesis nula para la hipótesis anteriormente mencionada. Es decir, que no hay diferencias significativas en cuanto al desarrollo de vocabulario mediante la lectura de un texto presentado en forma tradicional, en papel y el desarrollo de vocabulario mediante la lectura de un texto presentado a través del computador con estudiantes de segundo semestre de Inglés Técnico en el programa de Licenciatura en Informática, de la Universidad de Nariño. El valor crítico es de 0.05 ( $p<.05)$

\subsection{Variables}

Variable independiente

- Uso de materiales informáticos para lectura y vocabulario (Programa de reconstrucción de texto)

Variable dependiente

- Aprendizaje de vocabulario en Inglés

\subsection{Alcance del estudio}

Los resultados de este estudio y sus implicaciones permitirán tener una idea bastante inicial acerca de las ventajas y o desventajas del uso del computador como medio para complementar el aprendizaje de un idioma. Por otro lado, se obtendrá una evidencia inicial sobre la interacción de los usuarios entre sí y con la máquina, y sus reacciones: interés, motivación, atención, etc.

\section{REVISIÓN DE LA LITERATURA}

Hoy en día, a pesar de que este aspecto de la informática es bastante nuevo en nuestro medio y que en realidad la introducción de este tipo de tecnología educativa no ha tenido un rumbo aceptable a nivel de bachillerato, donde los computadores están siendo subutilizados y empleados en tareas completamente diferentes para lo que fueron diseñados, es importante manifestar la necesidad de realizar estudios de todo tipo desde el de observación hasta el experimental ya que los docentes tienen que concientizarse de que son los primeros y los más llamados a mirar qué pasa en el contexto educativo con la utilización de los computadores. Sobre todo en esta área que tantas expectativas ha estado creando en la gente y que todavía cuenta con muchos escépticos, entre ellos, los mismos docentes (Benavides, 1990). 
Revista de Investigaciones. Universidad de Nariño -UNED. Vol. VII, No. 1. (ISSN: 0121-120X). (pp. 71-77). 1995.

Es importante mencionar que a pesar de ser un campo nuevo y en el que usualmente se empieza a aplicar antes de investigar -lo cual parece ser una constante en todas las ciencias y artes- se han desarrollado algunos intentos por desarrollar estudios de carácter experimental en nuestro país. Henao y Giraldo (1991) en la Universidad de Antioquia realizaron uno de los primeros intentos en esta área, en nuestro medio, tratando de investigar la calidad y la cantidad de las composiciones escritas en español por niños entre 11 y 13 años utilizando los computadores como una herramienta de aprendizaje y un procesador de texto que les permitía desarrollar la habilidad de la escritura. Los sujetos tuvieron la oportunidad de mostrar diferencias significativas al producir sus textos en el computador a diferencia de lo que hicieron en forma manuscrita. Sus resultados son particularmente importantes puesto que muestran una evidencia más de la forma cómo los computadores pueden ser útiles, cómo pueden ayudar en el campo pedagógico convirtiéndose en una herramienta versátil, novedosa, interesante y productiva en tanto el participante pueda manipularlo y la máquina (en realidad, el programa) ponga en manos del sujeto diferentes posibilidades de aprendizaje. Benavides (1993) mediante un estudio similar, en campo de la lectura y vocabulario encontró que un grupo de estudiantes de nivel universitario mejoraron significativamente su nivel de velocidad de lectura mediante el uso de materiales informáticos. De la misma manera aunque no significativamente su nivel de aprendizaje de vocabulario también mejoró aunque en menor proporción (no significativamente a nivel estadístico).

Ha habido mucha especulación, sobre todo a nivel internacional sobre el uso de computadores en educación desde hace ya dos décadas. Muchos cuestionamientos se han hecho acerca de las ventajas del uso de los computadores en el aula de clase (Kinzer, 1986), sin embargo hay muchas expectativas acerca del efecto que estos puedan causar en cuanto a la atención y motivación en los estudiantes (White, 1983).

A nivel internacional, y a pesar de que se ha realizado investigación incipiente, aclarando que aún se necesita una buena estructuración de la literatura en este campo, la mayor parte de la evidencia acerca de la utilidad de los computadores como una forma alterna de enseñanza en educación ha sido anecdótica. Y a pesar de que en la opinión de los expertos y según algunos resultados de investigaciones pioneras, los esfuerzos se han orientado a promover el uso de los computadores en la enseñanza de la lectura y la escritura, muy pocos estudios se han realizado acerca de la diferencia entre la instrucción directa y el aprendizaje o desarrollo a que puede dar lugar la interacción del usuario con el computador, (Blair y Rupley 1986).

En estudios recientes las investigaciones se han concentrado en algunas de las implicaciones del uso de los computadores en el proceso de lectura (atención, terminación de tareas, y comprensión). Belmore $(1983,1985)$ encontró que los estudiantes de nivel universitario se demoraron más y comprendieron menos utilizando la presentación en el computador de la 
Revista de Investigaciones. Universidad de Nariño -UNED. Vol. VII, No. 1. (ISSN: 0121-120X). (pp. 71-77). 1995.

lectura a diferencia de lo que pudieron hacer sobre el papel. Zuk (1986) al trabajar con niños también encontró que la mayor diferencia en cuanto a la presentación del texto de lectura (texto en papel y texto en la pantalla del computador) radicaba en la terminación de la tarea de lectura. Los niños se demoraron más leyendo desde el computador que desde el papel. Sin embrago, Benavides (1993) en el campo de la lectura y vocabulario encontró que un grupo de estudiantes de nivel universitario mejoraron significativamente su nivel de velocidad de lectura mediante el uso de materiales informáticos y su nivel de aprendizaje de vocabulario también mejoró aunque en menor proporción (no significativamente a nivel estadístico).

En este estudio se abordará la pregunta de si en realidad las actividades de lectura generadas mediante ejercicios en el computador pueden incidir en el desarrollo de estrategias de aprendizaje de vocabulario. En otras palabras, el computador puede ayudar a desarrollar esta habilidad para hacer que el estudiante procese texto mediante su interacción con actividades de reconstrucción de texto?

\section{METODOLOGIA}

\subsection{Población y muestra}

Ya que se trata de un estudio de carácter semi-experimental piloto con el que se pretende tener una idea sobre la incidencia del uso del computador en variables como el desarrollo del vocabulario mediante la lectura, no hubo selección del grupo control, ni se seleccionó al grupo experimental randómicamente. Básicamente se utilizó una relación intra-sujetos en un trabajo de investigación en acción para la aplicación del tratamiento en un grupo de estudiantes de segundo semestre en la especialidad de informática de la Universidad de Nariño que tomaban clases de inglés técnico como parte del programa. El diseño del grupo experimental, de pruebas en serie, utiliza un grupo de 24 estudiantes de la Universidad de Nariño. En Benavides (1993) se había utilizado solo un grupo de 10 estudiantes, también de nivel universitario.

\subsection{Instrumentos}

El instrumento de medición incluía pretest y postest mediando un tratamiento basado en el trabajo con el computador parte del estudiante. Se realizaron 5 sesiones de trabajo para el tratamiento comprendidas en un período de seis semanas.

Este instrumento consistía en varios textos auténticos de Informática de aproximadamente 250 palabras y 18 líneas en los que se habían eliminado 20 términos que el estudiante tenía que suministrar una vez se finalizado el tratamiento en cada serie. Cinco de estos textos 
Revista de Investigaciones. Universidad de Nariño -UNED. Vol. VII, No. 1. (ISSN: 0121-120X). (pp. 71-77). 1995.

debían leerse en el formato tradicional de presentación, es decir sobre el papel, y los cincos restantes, a través del material informático presentado mediante el computador. Estos textos tenían aproximadamente el mismo índice de dificultad en la lectura (nivel preuniversitario (índice de Gunning's Fog $=10$ y Flesch $=74$ ) nivel de lectura adecuado para estudiantes de lengua extranjera.

Por razones de validez y confiabilidad lingüísticamente se han tenido en cuenta siempre el mismo número de términos de la misma parte del habla para que esto arroje resultados consistentes durante los diferentes tests. Es decir, se utilizaron como palabras FOCO un número determinado de verbos, adjetivos, nombres, pronombres, etc. Los respectivos tests se aplicaron después de cada sesión, tanto después de la presentación tradicional en papel como en la presentación con el computador.

\subsection{Materiales}

Los materiales que se utilizaron incluían hardware y software. El software incluía el programa QUARTEXT de Longman Editores que contiene cuatro diferentes módulos para la reconstrucción de texto tanto en forma tanto parcial como total, y en el que se ha enfatizado la lectura como medio para que los estudiantes los utilizaran en cada sesión. El disco contenía el sistema operativo DOS versión 4.1 y el programa QUARTEXT con los textos de lectura.

En cuanto al HARDWARE, se utilizaron los computadores de tipo IBM compatibles (AT) del aula de computadores de la Universidad de Nariño.

\subsection{Actividades}

El estudio inició con dos sesiones de una hora cada una en las que se introdujo a los estudiantes al uso y manejo del programa y de los cuatro módulos de reconstrucción del texto. Ya que los estudiantes tenían familiaridad con el manejo del computador no hubo necesidad de recalcar al respecto

Luego se utilizó el cronograma de actividades insistiendo en la absoluta necesidad de la asistencia de los estudiantes a las diferentes sesiones. Cada una de las sesiones incluía una al respecto.

Luego se realizó el cronograma de actividades insistiendo en la absoluta necesidad de la buena asistencia de los estudiantes a las diferentes sesiones. Cada una de las sesiones incluía una práctica con el programa QUARTEXT en el computador. 
Revista de Investigaciones. Universidad de Nariño -UNED. Vol. VII, No. 1. (ISSN: 0121-120X). (pp. 71-77). 1995.

\subsection{Tratamiento}

El tratamiento consistió en que los estudiantes tuvieran la oportunidad de leer un texto en el computador y para luego reconstruirlo completando 20 palabras que anteriormente se habían eliminado del texto.

En un primer módulo el programa creaba espacios en blanco dentro del texto para que el estudiante introdujera las palabras mediante selección múltiple o adivinara la misma escribiéndola en el teclado. Al mismo tiempo el computador brindó ayuda mediante el ofrecimiento de pistas dejando ver progresivamente cada letra de la palabra a medida que el estudiante oprimía la tecla espaciadora.

El programa llevaba el puntaje de aciertos, y de pistas ofrecidas, con el ingrediente de que a mayor número de pistas menor el puntaje del estudiante.

En el otro tipo de sesiones los estudiantes leían un texto de aproximadamente 250 palabras y discutían 20 palabras foco que se habían resaltado con anterioridad.

\subsection{Procedimiento}

El proceso o procedimiento en el tratamiento; uso de los materiales y aplicación del instrumento se realizó como se indica a continuación:

\subsubsection{CON EL COMPUTADOR}

1. los estudiantes iniciaban el computador y cargaban el sistema operativo que se proveía con el disco y luego el programa QUARTEXT.

2. Hecho esto, el estudiante tenía el tiempo disponible para leer el texto en la pantalla del computador y luego realizar el ejercicio de reconstrucción de texto.

3. El investigador instruyó a los estudiantes para que trabajaran en forma individual.

4. Después del trabajo con el computador, los estudiantes, realizaron una prueba de complementación del texto suministrando 20 palabras que el programa había eliminado de antemano.

\subsubsection{CON LA PRESENTACÓN EN EL PAPEL.}

1. Los estudiantes leían un texto completo de aproximadamente 250 palabras.

2. Los estudiantes comentaban acerca del significado de 20 palabras foco que habían sido resaltadas de antemano en el texto.

3. El investigador instruyó a los estudiantes para que trabajaron en forma individual 
Revista de Investigaciones. Universidad de Nariño -UNED. Vol. VII, No. 1. (ISSN: 0121-120X). (pp. 71-77). 1995.

4. Después del trabajo con el texto en el papel los estudiantes realizaron una prueba de complementación del texto suministrando las veinte (20) palabras foco que se habían eliminado de antemano.

\section{RESULTADOS}

Para el análisis de resultados se tuvieron en cuenta los promedios de los aciertos con los textos reconstruidos después de cada sesión tanto para los de la presentación con el computador como los de la presentación en papel.

\section{PROMEDIO DE ACIERTOS DE VOCABULARIO EN CINCO SESIONES}

\begin{tabular}{|c|c|c|}
\hline \multirow{2}{*}{$\begin{array}{c}\text { Estudiante } \\
\text { No. }\end{array}$} & \multicolumn{2}{|c|}{ Promedio de Aciertos $1 / 20$} \\
\hline & $\begin{array}{c}\text { Presentación } \\
\text { Normal }\end{array}$ & $\begin{array}{c}\text { Presentación en } \\
\text { el computador }\end{array}$ \\
\hline 1 & 5.6 & 6.4 \\
\hline 2 & 4.2 & 5.6 \\
\hline 3 & 7.4 & 7.8 \\
\hline 4 & 5.0 & 6.0 \\
\hline 5 & 7.0 & 7.4 \\
\hline 6 & 5.8 & 6.4 \\
\hline 7 & 7.0 & 7.6 \\
\hline 8 & 3.6 & 5.2 \\
\hline 9 & 5.4 & 6.2 \\
\hline 10 & 5.2 & 6.4 \\
\hline 11 & 5.8 & 6.4 \\
\hline 12 & 5.8 & 6.6 \\
\hline 13 & 4.8 & 6.2 \\
\hline 14 & 5.6 & 6.6 \\
\hline 15 & 4.8 & 5.8 \\
\hline 16 & 5.8 & 6.8 \\
\hline 17 & 5.8 & 6.6 \\
\hline 18 & 6.0 & 6.6 \\
\hline 19 & 5.6 & 6.6 \\
\hline 20 & 6.6 & 7.0 \\
\hline 21 & 4.6 & 5.8 \\
\hline 22 & 4.8 & 6.2 \\
\hline 23 & 7.2 & 7.6 \\
\hline 24 & 5.0 & 6.4 \\
\hline $\mathrm{X}$ & 5.6 & 6.5 \\
\hline
\end{tabular}

Tabla 1 Promedio de aciertos

Cabe anotar que los resultados de vocabulario en el cuadro anterior corresponden al promedio de las cinco sesiones tanto con los test en el papel como las cinco con el 
Revista de Investigaciones. Universidad de Nariño -UNED. Vol. VII, No. 1. (ISSN: 0121-120X). (pp. 71-77). 1995.

computador. El número en cada columna indica el promedio del número de palabras correctas de entre veinte (20) mediante el test de complementación.

En la gráfica siguiente (No. 1) se puede ver claramente las curvas de desempeño en cuanto al reconocimiento de vocabulario promediando las dos clases de aciertos con cada una de las presentaciones del texto (en el papel y en el computador) de los veinticuatro estudiantes del grupo. Las ganancias en cuanto a aprendizaje de vocabulario son importantes hasta el punto de poderlo determinar gráficamente.

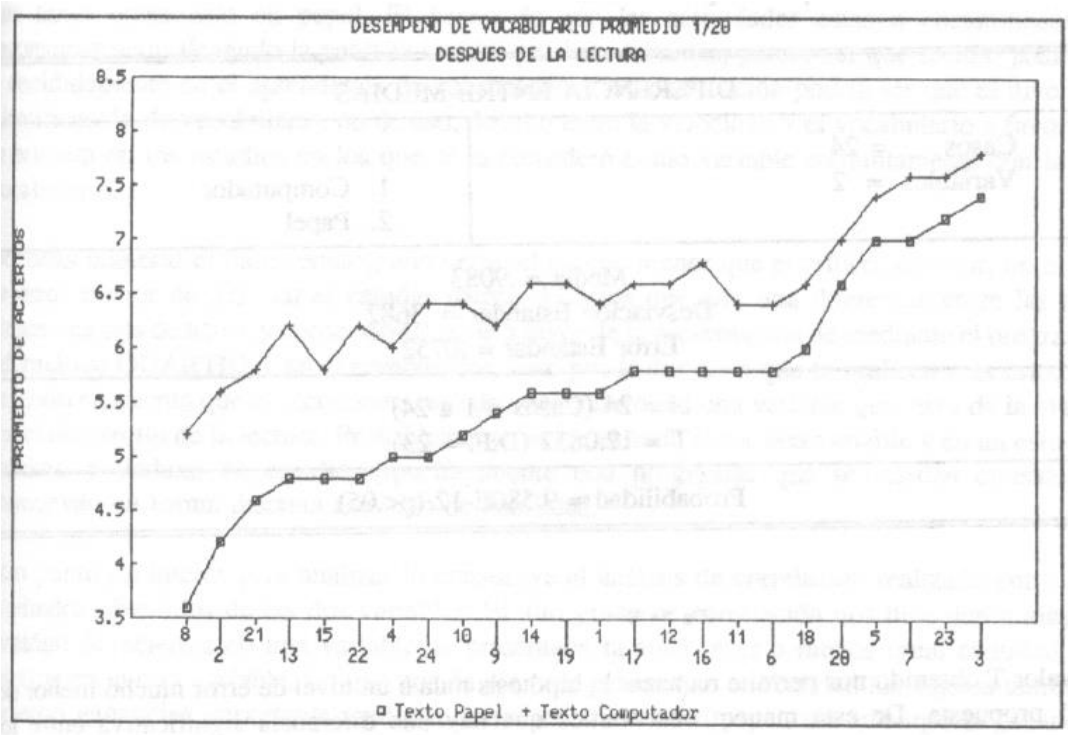

Gráfica 1 Desempeño de vocabulario

\subsection{Validación de hipótesis}

Para la validación de hipótesis se utilizó el procedimiento estadístico de T- test de pares que ofrece una buena confiabilidad en estudios de este tipo con grupos de muestra pequeños (en este caso $\mathrm{N}=24$ ).

A continuación se puede observar los resultados en cuanto a valor $T$ con D.F. $=23$, con la aclaración que se comparan valores en pares, de cada uno de los estudiantes en los test tomando el valor como promedio de aciertos de las cinco sesiones en cada caso del tratamiento. 
Revista de Investigaciones. Universidad de Nariño -UNED. Vol. VII, No. 1. (ISSN: 0121-120X). (pp. 71-77). 1995.

\begin{tabular}{|c|c|c|}
\hline \multicolumn{3}{|c|}{ DIFERENCIA ENTRE MEDIAS } \\
\hline $\begin{array}{l}\text { Casos }= \\
\text { Variables }=\end{array}$ & $\begin{array}{r}=24 \\
=2\end{array}$ & $\begin{array}{l}\text { 1. Computador } \\
\text { 2. Papel }\end{array}$ \\
\hline \multicolumn{3}{|c|}{$\begin{array}{c}\text { Media }=.9083 \\
\text { Desviación Estandar }=.3682 \\
\text { Error Estandar }=.0752\end{array}$} \\
\hline \multicolumn{3}{|c|}{$\begin{array}{l}\mathrm{N}=24(\text { Casos }=1 \text { a } 24) \\
\mathrm{T}=12.0852(\mathrm{D} . \mathrm{F} .=23)\end{array}$} \\
\hline \multicolumn{3}{|c|}{ Probabilidad $=9.580 \mathrm{E}-12(\mathrm{p}<.05)$} \\
\hline
\end{tabular}

Tabla 2 Prueba de hipótesis; Valor T; Diferencia de medias

En la Tabla 2 el valor T obtenido permite rechazar la hipótesis nula a un nivel de error mucho menor del 0.05 propuesta. De esta manera, se observa que hay una diferencia significativa entre los promedios de los dos test y que la variable independiente ciertamente estaría incidiendo significativamente sobre la dependiente.

También es importante considerar el grado de correlación entre las dos variables, aprendizaje de vocabulario por medio de la presentación en el computador y mediante la presentación en papel. Se puede observar en la tabla 2 que la correlación de los dos resultados es bastante alta y significativa $(r=0.97)$ con una probabilidad de error bastante baja $(p<0.5)$.

\subsection{Discusión}

Este estudio arroja un resultado e cierto modo diferente con los encontrados en Belmore (1986), Zuk (1986) y Benavides (1993), en cuanto al desarrollo de vocabulario a través de actividades realizadas en el computador. Tal vez, el énfasis de estos estudios se centraba en las dos variables: velocidad y comprensión de vocabulario y sobre todo en ele de la velocidad a diferencia del presente estudio en el cual solo de observó y midió la variable aprendizaje de vocabulario. Si el computador puede servir para que se aprenda más sin afectar la comprensión (controlada mediante los ejercicios de vocabulario) entonces el proceso de lectura puede en cierta forma no ser totalmente independiente del modo de presentación, incidiendo directamente sobre el aprendizaje de vocabulario.

Es necesario aclarar que los resultados de vocabulario (reconocimiento) en este estudio contrastan con los anteriores en cuanto que en este caso se puede mostrar una diferencia significativa mediante los test a favor de las ganancias logradas en la presentación del texto con el computador que con el texto presentado en papel. El hecho de que las actividades estaban encaminadas a reconstruir un texto llenando la pantalla con las palabras faltantes, al 
Revista de Investigaciones. Universidad de Nariño -UNED. Vol. VII, No. 1. (ISSN: 0121-120X). (pp. 71-77). 1995.

parecer fue positiva y marcó decididamente el aprendizaje de vocabulario. Otra explicación podría ser que el nivel de identificación de vocabulario, no de uso, decidió entre la velocidad y el vocabulario a favor de la primera en los estudios en los que se la considero como variable conjuntamente con la de vocabulario.

De todas maneras, el haber conseguido un nivel de probabilidad mucho menor que el crítico, es decir, un nivel de error menor de 0.5 en el estudio parece indicar que hay una diferencia entre las dos presentaciones de texto, y consecuentemente a favor de la presentación del texto mediante el programa informático QUARTEXT en el computador. Esto puede dar pie a que se realicen más estudios teniendo en cuenta que el reconocimiento de vocabulario pueda considerarse una variable que vaya de la mano con el desarrollo de la lectura. Probablemente, se necesitaría aislar esta variable y en un estudio próximo dedicar su estudio específicamente con programas que se puedan considerar promuevan en formar discreta este tipo de habilidad.

Otro punto de interés para analizar lo constituye el análisis de correlación realizado entre los resultados obtenidos de las dos variables. El alto grado de correlación indica que a mayor cantidad de aciertos en una variable se presentaba también aproximadamente igual cantidad de aciertos en la otra variable. Lo que da pie para pensar que en realidad los sujetos también hicieron ganancias importantes en vocabulario mediante la presentación en el papel, sin embargo la ganancia fue mayor significativamente en la presentación en el computador.

La tabla No 1 también indica que los sujetos que hicieron menos aciertos mediante la presentación en papel hicieron mayores ganancias en aciertos en la presentación del computador. Esto puede corroborar el presupuesto de que a menor nivel de conocimiento o desarrollo de una habilidad, dos sujetos en igualdad de condiciones, tiene mayores posibilidades de ganancias en cuanto a aprendizaje el de menor nivel que el de mayor nivel de conocimientos en la misma área.

\subsection{Resultados de la observación}

En primer lugar, la novedad de las actividades en las que los sujetos se vieron comprometidos fue un factor importante para el éxito del estudio, ya que no se notó ninguna clase de cansancio o pérdida de la motivación. Al contrario, las sesiones se extendían aproximadamente por unos diez a quince minutos más después de terminado el tiempo asignado. Además, los sujetos demostraban una atención muy alta puesto que querían saber todo lo que ocurría en la pantalla, preguntando al profesor o recurriendo a la ayuda mediante el teclado del computador.

En segundo lugar, es importante destacar la interacción de los sujetos con el computador la cual iba en aumento a medida que pasaban las sesiones. La intervención del investigador 
Revista de Investigaciones. Universidad de Nariño -UNED. Vol. VII, No. 1. (ISSN: 0121-120X). (pp. 71-77). 1995.

como guía en las tres primeras sesiones se redujo al mínimo en las finales puesto que los sujetos hacían gala de sus propias aptitudes para solventar cualquier situación. Por ejemplo, en el caso de que algo no funcionara bien tenían que oprimir determinada tecla ('ayuda'), de la misma manera cuando se deseaba que el computador "supiera" que tenía que dar una respuesta. Sin embrago, en algunas ocasiones, sobre todo al final, algunos sujetos hicieron cosas que no estaban programadas para el estudio como salirse del programa o ingresar en otros módulos, y tener acceso a diferentes textos. Esto, es un índice de la facilidad con que el estudiante es inducido a intentar algo, mediante el teclado, con la certeza de que el computador tendría que responder algo. El solo hecho de saber "qué pasaría si..." tiene muy buen indicio en este contexto educativo, sobre todo cuando el énfasis es el aprendizaje más no de instrucción directa o explícita. Muchas de estas cosas no se había previsto, sin embargo los sujetos parecían intuir lo que había pasado al realizar algunas incursiones esporádicas en el programa.

Por último, a pesar de que a los sujetos se les dijo que podían consultar con sus compañeros tanto en el trabajo de la lectura mediante el papel y con el computador (trabajando dos con un solo computador) fue importante la interacción de los sujetos entre sí. Unos preguntaban a otros, y algunos se detenían a observar la pantalla del computador de los compañeros para ver, no siempre las respuestas, sino la forma cómo lo hacían, especialmente durante las dos primeras sesiones de trabajo con el computador. Sin embargo, durante las tres últimas sesiones, los estudiantes si observaban el resultado o puntaje (mediante el porcentaje de aciertos) que tenía cada pareja, ya que aparecía en la parte superior de la pantalla del computador, en diferentes ocasiones. Según los estudiantes, lo hacían para tener un punto de referencia de cómo iban demás compañeros en cuanto a la cantidad de texto completado. Esto, por supuesto puede dar una idea del factor de competitividad que ofrecía este tipo de actividades.

\section{CONCLUSIONES E IMPLICACIONES}

En este aspecto de la lectura y el vocabulario, que es el tópico de este estudio se puede ver cómo una actividad en la que el estudiante se enfrenta a un problema en el que principalmente tenía que procesar diferentes textos para poder reconstruirlos, como meta, se puede utilizar para el desarrollo del aprendizaje de vocabulario mediante el computador. Esto significaría que esta tradicional, e inclusive lograr mejoría en el aprendizaje de vocabulario. Una alternativa al texto en el sentido de que otras actividades, no necesariamente más complejas, diferentes a las de solo leer, pueden ser aprovechables mediante el uso del computador.

Otro aspecto especial consideración en este estudio a nivel pedagógico, el cual destaca más el papel del computador como una herramienta de aprendizaje, es la prioridad por la cual el estudiante tiene contacto directo con el computador y se puede beneficiar mediante su 
Revista de Investigaciones. Universidad de Nariño -UNED. Vol. VII, No. 1. (ISSN: 0121-120X). (pp. 71-77). 1995.

interacción con los programas a través de este en cuanto lo que se logra se produce en el proceso. Esto permitiría que el estudiante permanezca más tiempo en exposición e interacción directa con el computador para efectos de trabajar (manipular y utilizar) la información pertinente a la actividad en cuestión manejando su propio ritmo de aprendizaje y desarrollando sus propias estrategias. Sería un error pues, considerar que el computador esté en este caso realizando un trabajo como el del profesor o instructor ya que la máquina no enseña directamente algo, sin embargo permite al estudiante descubrir, manejar, y utilizar a sus acomodo la información siempre y cuando la actividad sea de este tipopedagógico- (programas o software educativos).

Esto permite reconocer y enfatizar la importancia de que los docentes se inicien en el conocimiento de los materiales informáticos, es decir, diferentes tipos de programas para que los puedan aplicar, evaluar, realizar investigación y aportar la parte creativa. En otras palabras, es importante considerar que el papel del docente tendrá que cambiar sustancialmente cuanto este se integre a un proceso en el cual el estudiante se beneficiará más de una instrucción complementaria que le permitirá eventualmente aprender más de una y mejor. Se podría estar acercando, entonces al hecho de que el profesor en el inmediato futuro tiene que empezar a cambiar su papel en el proceso educativo. En otras palabras se tendría que considerar un profesor o pedagogo con otro tipo de ingredientes y cualidades derivadas de la introducción de la tecnología educativa.

Probablemente, en el futuro cercano, no será raro ni atrevido que el profesor sea un analista de sistemas educativos, o un programador o diseñador de programas informáticos educativos, que a la postre le permitirá al usuario utilizar lo más adecuado para desarrollar cualquier tipo de habilidad e integrar cualquier tipo de conocimiento en forma más productiva.

Por último, es necesario mencionar que este estudio trató de abordaje y comprender a nivel exploratorio una de las utilidades del uso del computador en Educación, más concretamente en el aprendizaje de los Idiomas. No se pretende generalizar estos resultados ni concluir acerca de la todavía incipiente aplicación de la Informática Educativa, sino manifestar que se necesitan más y diferentes tipos de estudios que aborden tópicos variados en este campo, controlado en forma más eficiente las variables en cuestión. Solo así se podría acercar a la comprensión de cómo utilizar mejor la tecnología que brinda nuestra época aplicada a la Educación que cada día que pasa se hace más necesaria tanto para docentes como para los estudiantes en los diferentes campos del saber.

Una de las conclusiones más importantes de este estudio se relaciona con la utilidad del computador como herramienta de aprendizaje. Es necesario establecer esta diferencia entre instrucción y aprendizaje ya que en el computador subyace la esencia del pedagogo que guía 
Revista de Investigaciones. Universidad de Nariño -UNED. Vol. VII, No. 1. (ISSN: 0121-120X). (pp. 71-77). 1995.

y ayuda, crea, incentiva, simula y permite que el estudiante desarrolle sus propias estrategias de aprendizaje, no necesariamente en el sentido de una instrucción directa sino como un medio para aprender mejor. Si el computador se considerara que hace las veces de un instructor, entonces sería mejor olvidarse del asunto puesto que esto no presenta ninguna clase de innovación. Precisamente, si se tiene al computador como una herramienta de aprendizaje para el estudiante, y de apoyo para el profesor por el hecho de ser mucho más versátil, manipulable, e interesante como material de aprendizaje y desarrollo adicional al texto, por ejemplo, es precisamente por el carácter de que su aplicación puede contener aspectos específicos y diferentes a la enseñanza explícita en cuanto su interacción con el usuario y promueva nuevas estrategias de aprendizaje y procesos cognitivos.

Finalmente, deseo agradecer, a los estudiantes que participaron como sujetos de este estudio de investigación en la acción sin los cuales no habría sido posible realizar ningún tipo de actividad investigativa interesante. También, mis agradecimientos tanto al director de la sala de informática de turno quien dedicó el interés necesario en este campo, así como a la Universidad de Nariño.

\section{Bibliografía}

Belmore, S. (1985). Reading Computer-presented Text. Bulletin of the Psychonomic Society. 23 (1), $12^{\prime} 14$.

Benavides, B. Jorge E. (1993). Informática educativa para la enseñanza de los Idiomas. Revista de Investigaciones. Universidad de Nariño. Año7, No. 1, vol. VI. 196-206.

Benavides, B. Jorge E. (1989). Iniciación a la Instrucción Complementaria del Inglés mediante Computadores. Revista de Investigaciones, Universidad de Nariño. No. 5, vol. III. 179-193.

Benavides, B. Jorge E. (1993). Desarrollo de la velocidad de lectura y vocabulario mediante el uso de materiales informáticos. Revista de Investigaciones "Criterios". Universidad Mariana. (En imprenta)

Blair, F., Rupley, W. \& Jones, M. (1986). Microcomputers: Another false Prophet? Reading Research and Instruction. 26 (1), 58-61.

Henao, Octavio, \& Luz Giraldo. (1991). El computador en la enseñanza de la escritura. Medellín (Universidad de Antioquia, Facultad de Educación). Textos y Diseños.

Kinzer, Ch. (1986). A 5 Part Categorization for use of microcomputers in Reading Classrooms. Journal of Reading. 30 (3). 56-76.

White, M. (1983). Synthesis of Research on Electronic Learning. Educational Leadership. 40(8), 13-15.

Zuk, D. (1986). The effects of Microcomputers on Children`s attention to Reading. Computers in the Schools. (3), 39-51. 